\title{
CATETERISMO URINÁRIO: FACILIDADES E DIFICULDADES RELACIONADAS À SUA PADRONIZAÇÃO
}

\author{
Alessandra Mazzo1, Simone Godoy², Leila Marchi Alves³, Isabel Amélia Costa Mendes", Maria Auxiliadora \\ Trevizan ${ }^{5}$, Elaine Maria Leite Rangel ${ }^{6}$
}

${ }^{1}$ Doutor em Enfermagem. Professor Doutor do Departamento de Enfermagem Geral e Especializada (DEGE) da Escola
de Enfermagem de Ribeirão Preto (EERP) da Universidade de São Paulo (USP). Centro Colaborador da OMS para o
desenvolvimento da Pesquisa em Enfermagem. São Paulo, Brasil. E-mail: amazzo@eerp.usp.br
2 Doutor em Ciências. Especialista em Laboratório do DEGE/EERP/USP. São Paulo, Brasil. E-mail: sig@eerp.usp.br
${ }^{3}$ Doutor em Enfermagem. Professor Doutor do DEGE/EERP/USP. São Paulo, Brasil. E-mail: lmarchi@eerp.usp
${ }^{4}$ Doutor em Enfermagem. Professor Titular do DEGE/EERP/USP. São Paulo, Brasil. E-mail: iamendes@eerp.usp.br
${ }^{5}$ Doutor em Enfermagem. Professor Titular aposentada e vinculada ao Programa Enfermagem Fundamental do DEGE/EERP/
USP. São Paulo, Brasil. E-mail: trevizan@eerp.usp.br
${ }^{6}$ Doutor em Enfermagem. Professora Adjunto I do Departamento de Enfermagem do Centro de Ciências da Saúde da
Universidade Federal do Piauí. Piauí, Brasil. E-mail: elairgel@gmail.com

RESUMO: Pesquisa exploratória e descritiva para verificar a existência e fatores relacionados a padronização do cateterismo urinário, em uma cidade do interior do estado de São Paulo, Brasil. Os dados foram coletados por meio de entrevista, mediante aprovação do Comitê de Ética em Pesquisa da Escola de Enfermagem de Ribeirão Preto. Das nove instituições hospitalares entrevistadas, 100\% possuem o procedimento de cateterismo urinário padronizado. As instituições apresentam diferenças nos procedimentos propostos, o que interfere de maneira positiva e negativa na sua padronização e coloca em dúvida se o padronizado é o que está sendo realizado. Conclui-se que existe a necessidade de se reavaliar a padronização proposta, com discussões interdisciplinares, verificar se o proposto está sendo realizado e buscar estratégias de treinamento e ensino passíveis de aplicação.

DESCRITORES: Cateterismo urinário. Métodos. Enfermagem. Protocolos.

\section{URINARY CATHETERIZATION: FACILITIES AND DIFFICULTIES RELATED TO ITS STANDARDIZATION}

\begin{abstract}
This descriptive and exploratory study aimed to verify the existence of factors related to the standardization of urinary catheters in a city in the interior of the state of São Paulo, Brazil. Data was collected through interviews after approval obtained from the Research Ethics Committee of the University of São Paulo at Ribeirão Preto School of Nursing. Of the nine hospital institutions who took part in the study, $100 \%$ have a standardized procedure for urinary catheterization. The institutions present differences in the proposed procedure, which interferes positively and negatively in its standardization and places doubt upon whether the standardized procedure is indeed being carried out. This study concludes that there is a need to reevaluate the proposed standardization, with interdisciplinary discussions to verify if the proposal is being carried out and to search for applicable training and teaching strategies.
\end{abstract}

DESCRIPTORS: Urinary catheterization. Methods. Nursing. Protocols.

\section{LA CATETERIZACIÓN URINARIA: FACILIDADES Y DIFICULTADES RELACIONADAS CON SU ESTANDARIZACIÓN}

\begin{abstract}
RESUMEN: Estudio exploratorio y descriptivo con objeto de determinar qué factores están relacionados con la estandarización de los catéteres urinarios, en una ciudad del interior del estado de São Paulo, Brasil. El estudio fue realizado a través de entrevistas, mediante aprobación del Comité de Ética en Investigación de la Escuela de Enfermería de Ribeirão Preto. De las nueve instituciones hospitalarias entrevistadas, $100 \%$ posee un procedimiento de cateterización vesical estandarizado. Las instituciones presentan diferencias en el procedimiento propuesto, lo que interfiere de manera positiva y negativa en su estandarización y pone en duda si el estandarizado es el que está siendo realizado. Se concluye que existe la necesidad de reevaluar la estandarización propuesta, con discusiones interdisciplinarias, verificar si el propuesto está siendo realizado y buscar estrategias de entrenamiento y enseñanza pasibles de aplicación.
\end{abstract}

DESCRIPTORES: Cateterismo urinário. Métodos. Enfermería. Protocolos. 


\section{INTRODUÇÃO}

O enfermeiro desempenha importante atividade na assistência de enfermagem em relação às eliminações urinárias, desenvolvendo ações que vão da promoção da saúde aos cuidados agudos. Nas ações voltadas à promoção da saúde, o enfermeiro desenvolve processos de educação e atua promovendo o balanço hídrico adequado, a prevenção de infecções do trato urinário, os cuidados com exercícios perineais, higiene e obesidade. Aos clientes que apresentam a função urinária alterada, medidas de intervenção em enfermagem se fazem necessárias, dentre as quais o cateterismo urinário. $^{1-3}$

O cateterismo urinário é um procedimento amplamente utilizado, sendo de inestimável valor ao tratamento de processos patológicos. Seu uso direciona-se a pacientes que apresentam incontinência urinária, retenção urinária, quando se faz necessária a avaliação exata do débito urinário, restrições pós-operatórias, coleta de amostras de urina, irrigação de bexiga ou instilação de medicamentos e nas cirurgias urológicas. ${ }^{1,4}$

Cerca de $10 \%$ dos pacientes hospitalizados são expostos ao cateterismo urinário. O procedimento de inserção do cateter é estéril e sua complicação mais frequente é a infecção urinária. A Infecção do Trato Urinário (ITU) representa cerca de $40 \%$ das infecções hospitalares e resulta em repercussão econômica, sequelas, complicações e danos imensuráveis à população. ${ }^{5-7}$

No cateterismo urinário de alívio e intermitente (realizado em intervalos rotineiros), as sondas são retiradas logo após o esvaziamento da bexiga, o que implica em menores taxas de infecção de trato urinário. No cateterismo urinário de demora o risco para infecção se torna maior após 72 horas de permanência com o cateter, e pode ser agravado pelo trauma do tecido uretral na sua inserção. ${ }^{4,7-8}$

A inserção do cateter urinário pode ainda ocasionar outras complicações, como traumatismo uretral, dor e falso trajeto, possibilitando, quando associada a diferentes fatores e na variância do tempo de cateterização, a instalação de infecção em outro sítio do organismo, a litíase urinária renal e vesical, uretrite, periuroretrite e abscesso periuroretral, divertículo uretral, fístula uretral, prostatite, epidimite, necrose peniana e câncer de bexiga. ${ }^{4}$

Conforme a Lei $n^{0} 7.498 / 1986$, que dispõe sobre o Exercício Profissional da Enfermagem, o enfermeiro e o técnico de enfermagem são os profissionais habilitados à realização deste procedimento. ${ }^{9}$ No entanto, ao inserirem o cateter vesical, na prática clínica, esses profissionais utilizam, na maior parte do tempo, exclusivamente o seu conhecimento prático, ocasionando situações que colocam em risco os pacientes e os próprios profissionais. ${ }^{10-11}$

Ao participarem de processos de desenvolvimento e adoção de políticas de qualidade e acreditação, as instituições de saúde elaboram protocolos assistenciais, com embasamento científico e determinação das atribuições dos profissionais. Não há dúvida que esse é um processo que gera ganhos, direcionando e subsidiando as atividades de enfermagem. ${ }^{10-12}$

$\mathrm{Na}$ literatura, as técnicas de introdução e manutenção do cateter urinário são descritas de diferentes maneiras e, apesar de as recomendações de prevenção de infecções relacionadas à introdução e manutenção do cateter urinário terem sido atualizadas recentemente, ainda não há consenso em diversas ações que compreendem alguns passos do procedimento, como por exemplo o uso dos antissépticos, lubrificantes, dentre outros, podendo ocasionar na elaboração dos protocolos institucionais relacionados ao cateterismo urinário diversas interpretações e subjetividade. . $^{1,2,4-6}$

Uma vez que a realização e o acompanhamento do cateterismo urinário é parte do trabalho da enfermagem e fator de manutenção da segurança e garantia da qualidade da assistência prestada ao paciente, questionamos como tal intervenção tem sido realizada na prática clínica da profissão. Para tanto, este estudo procurou verificar a existência e fatores relacionados a padronização do cateterismo urinário e caracterizar as instituições que a utilizam.

\section{METODOLOGIA}

Estudo de abordagem quantitativa de natureza descritiva, desenvolvido junto às instituições hospitalares em uma cidade do interior do estado de São Paulo, mediante aprovação do Comitê de Ética em Pesquisa da Escola de Enfermagem de Ribeirão Preto - USP, conforme Resolução ${ }^{\circ}$ 196/96 da Comissão Nacional de Pesquisa (Processo 1.005/2009). Assegurada a liberdade de participação e o anonimato dos participantes.

Das treze instituições hospitalares do município (dez hospitais gerais, duas maternidades 
e um hospital secundário), nove consentiram na realização do estudo.

Os dados foram coletados pelos próprios pesquisadores, por meio de entrevista semiestruturada, após formalização da visita no serviço. Foi entrevistado o enfermeiro representante da instituição, designado pelo responsável institucional. O formulário utilizado na coleta de dados foi composto de dados demográficos e de identificação da instituição e do profissional entrevistado e do estabelecimento da positividade ou não em relação à padronização da intervenção de cateterismo vesical na instituição. Quando obtida resposta afirmativa quanto à padronização da intervenção, questionou-se o entrevistado sobre: tempo e responsabilidade pela elaboração, tempo de aplicação, descrição e fatores de interferência, segundo sua opinião.

As instituições foram classificadas, quanto ao porte, em pequeno, médio ou grande porte; e o regime de financiamento foi classificado em público, privado ou misto. ${ }^{13}$

A análise dos dados das entrevistas foi realizada no Microsoft Excel, por meio de análise de frequência e porcentagem e de acordo com a literatura sobre o assunto; posteriormente, os dados foram apresentados em forma de relatório discursivo.

\section{RESULTADOS E DISCUSSÕES}

Dos 13 hospitais do município, nove concordaram em participar do estudo, dos quais cinco (55,6\%) são privados, três $(33,3 \%)$ públicos e um $(11,1 \%)$ filantrópico. Duas $(22,2 \%)$ instituições eram de pequeno porte, quatro $(44,4 \%)$ de médio porte e três $(33,3 \%)$ de grande porte, sendo que a média de ocupação entre os hospitais privados era de 10,1 pacientes/leitos/mês, entre os hospitais públicos de 6,5 pacientes/leito/mês e no hospital misto foi de 3,3 pacientes/leito/mês.

Todos os serviços entrevistados possuem padronização do procedimento de cateterismo urinário. Para estabelecer e adotar o protocolo do procedimento, as instituições levaram de seis a 50 meses, com média de tempo de 26,4 meses. Em três $(33,3 \%)$ houve um intervalo de tempo entre o estabelecimento do protocolo e sua adoção na prática clínica. Dentre essas, duas (22,2\%) apresentam como intervalo entre o estabelecimento e a adoção do protocolo 12 meses, e uma (11,1\%) 10 meses. Foi considerada como padronização do procedimento de cateterismo urinário a existência de publicação interna de protocolo institucional e como a adoção do procedimento o treinamento de toda a equipe e a acessibilidade à sua realização.

Os enfermeiros entrevistados foram responsáveis pelo estabelecimento e adoção na prática clínica do protocolo de cateterismo urinário; desses, quatro $(44,4 \%)$ eram enfermeiros do Serviço de Educação Continuada, dois $(22,2 \%)$ eram enfermeiros da Unidade de Terapia Intensiva (UTI), dois $(2,22 \%)$ eram enfermeiros do Serviço de Controle de Infecção Hospitalar (SCIH) e um $(11,1 \%)$ era enfermeiro da Clínica de Internação.

Nas nove instituições foi mencionado que o procedimento de cateterismo urinário é realizado pelo enfermeiro, pela equipe de enfermagem e pela equipe médica, após prescrição médica. É também a prescrição médica a determinante da suspensão do uso do cateter urinário.

Observa-se que é efetiva a participação do enfermeiro na elaboração e adoção dos protocolos de cateterismo urinário, assim como na realização do procedimento em todas as instituições pesquisadas. No entanto, ao realizar esse procedimento com base na prática clínica, o enfermeiro e a equipe de enfermagem aumentam as possibilidades de erros, colocando em risco a segurança e a qualidade do cuidado. ${ }^{10-14}$

Diferentes formas de conduta profissional, ocasionadas por atos técnicos repetidos, são incapazes de reproduzir os mesmos resultados, uma vez que são executadas por indivíduos a outros indivíduos, em diferentes contextos de trabalho, não contemplando a racionalização das rotinas e a segurança da assistência de enfermagem. . $^{10,14-15}$ No intuito de resolução e mudança nesses complexos processos assistenciais e com a necessidade de assegurar e qualificar a assistência de enfermagem prestada ao paciente, propostas de melhora da comunicação nas ações em equipe de trabalho em saúde e incentivos a programas de acreditação de organizações de saúde, com a qualificação profissional e o aumento da força de trabalho em enfermagem, fazem parte de estratégias estabelecidas. Dentre elas, a padronização dos procedimentos traz orientação aos questionamentos, esclarece condutas, deve ser construída embasada em diretrizes e normas institucionais e ser seguida pelos profissionais de saúde de uma mesma instituição. ${ }^{12,15-16}$

A padronização dos processos tem se mostrado uma importante ferramenta dentro de um modelo de sistema gerencial, no oferecimento de um serviço qualitativo ao paciente, contribuin- 
do com a implementação de novas tecnologias, melhoria da assistência e satisfação da equipe e está relacionada a padrões de cuidado, voltados aos direitos do paciente em receber assistência de acordo com suas necessidades. ${ }^{15}$

Com relação à sequência do procedimento de cateterismo urinário padronizado descrito nas entrevistas, em oito $(88,9 \%)$ instituições ela é composta pelo preparo do material, interação enfermeiro/paciente, higiene externa, disposição e abertura do material junto ao paciente, montagem do material utilizado (conexão do cateter vesical junto ao coletor-sistema fechado), antissepsia, cateterização, fixação do cateter e coletor, recomposição da unidade do paciente. Dentre os profissionais entrevistados, um $(11,1 \%)$ não fez referência à higiene externa prévia à antissepsia, seguindo, contudo, as demais etapas descritas pelas outras instituições. A operacionalização das etapas do procedimento foi apresentada de diferentes maneiras em todas as instituições pesquisadas. No entanto, observa-se que a sequência preconizada para a sua realização foi descrita de acordo com o encontrado nos livros-textos e nas diretrizes que fundamentam a prática de enfermagem sobre o assunto, porém com particularidades e diferenças no "como fazer" ., $, 6,17-18$

Com relação ao registro no prontuário no fim do procedimento, ele foi mencionado por cinco $(55,5 \%)$ das instituições pesquisadas. Três $(33,3 \%)$ referem que, além do registro em prontuário, anotam, no próprio coletor urinário, dados referentes à data de inserção do cateter e dados de identificação do profissional responsável pela cateterização urinária (nome, função e identificação junto ao Conselho Regional de Enfermagem). Dentre essas, duas $(22,2 \%)$ utilizam para essa anotação uma etiqueta autoadesiva padronizada no serviço, que é colada na bolsa coletora de urina. O registro de enfermagem é amparo legal do profissional, proporciona a continuidade da assistência em enfermagem prestada ao paciente e constitui-se em fator de extrema relevância no exercício da profissão. ${ }^{1-2,6,12}$.

$\mathrm{Na}$ necessidade de troca do cateter vesical, cinco $(55,6 \%)$ instituições declararam que a decisão de troca do cateter é tomada em conjunto entre os profissionais da enfermagem e o médico, três $(33,3 \%)$ em consenso entre o enfermeiro, médico e Serviço de Controle de Infecção Hospitalar e em uma $(11,1 \%)$ declarou que cabe somente ao médico a decisão da troca do cateter.
Ao descreverem o protocolo de troca do cateter, todas as instituições pesquisadas referem que realizam uma nova cateterização urinária, com a substituição de todo o material, quando necessário. Em duas (22,2\%) instituições salientou-se que, quando o cateter é inserido em procedimento médico-cirúrgico e há necessidade de troca ou desobstrução do sistema coletor, é realizada a desconexão entre a sonda e o coletor urinário, optando-se somente pela troca do segundo, não sendo esta conduta descrita no protocolo padronizado, o que impossibilita o seu registro, impede o seu rastreamento, amplia os riscos e não assegura ou garante a qualidade do atendimento ao paciente. .,17,19

Os motivos relacionados para a troca do sistema e inserção de novo cateter nas instituições foram ITU (2-11,7\%), desconexão do sistema coletor $(2-11,7 \%)$, problemas com o coletor de urina $(2-11,7 \%)$, mudanças nas características da diurese $(2-11,7 \%)$, obstrução do cateter $(2-11,7 \%)$, a alta do paciente para a residência portando o cateter (1-5,9\%), internação de paciente de unidade hospitalar ou ambulatorial externa ao serviço portando cateter vesical (1-5,9\%), contaminação na técnica de instalação ou manutenção do cateter (1-5,9\%), desconexão acidental do cateter (1-5,9\%), mau funcionamento do cateter e (1-5,9\%) febre desconhecida (1-5,9\%). Embora quase todos os fatores mencionados estejam acordados com as diretrizes e literatura que sustentam o assunto, com exceção da possibilidade da troca do cateter vesical em atendimento às características ou necessidades do serviço, merece destaque a forma aleatória e individual como foram citados entre as instituições. ${ }^{6,17}$

Ainda que o procedimento de cateterismo vesical venha sendo salientado há mais de uma década nas diretrizes internacionais, sua padronização e execução envolvem componentes do contexto prático onde é realizado. Para que ocorra de maneira adequada é necessário o envolvimento de todos os profissionais e da instituição. Nesse sentido, recursos físicos, materiais, humanos e processos administrativos das instituições têm direcionado de maneira positiva ou negativa o processo de adoção e manutenção dos protocolos padronizados. ${ }^{6,10,14}$

Os dados relacionados às facilidades e dificuldades relatadas em relação à padronização do procedimento estão apresentados na tabela 1 e na tabela 2. 
Tabela 1 - Distribuição de frequência e percentual das facilidades relacionadas à padronização do procedimento de cateterismo vesical, segundo instituições públicas, privadas e mista. Ribeirão Preto-SP, 2009

\begin{tabular}{lccccc}
\hline \multirow{2}{*}{ Facilidades } & \multicolumn{3}{c}{ Instituições } & \multicolumn{2}{c}{ Total } \\
\cline { 2 - 4 } & Públicas & Privadas & Mista & n & \% \\
\hline $\begin{array}{l}\text { Treinamento de todos os funcionários } \\
\text { recém-admitidos na enfermagem }\end{array}$ & 1 & - & - & 1 & 20,0 \\
$\begin{array}{l}\text { Reconhecimento do trabalho da enfermagem perante as demais equipes } \\
\text { de saúde }\end{array}$ & 1 & - & - & 1 & 20,0 \\
Participação da equipe de enfermagem nos treinamentos & 1 & - & - & 1 & 20,0 \\
Padronização dos materiais utilizados pela instituição & - & 1 & - & 1 & 20,0 \\
Comodidade no cuidado por parte do paciente e equipe de enfermagem & - & - & 1 & 1 & 20,0 \\
\hline Total & $\mathbf{3}$ & $\mathbf{1}$ & $\mathbf{1}$ & $\mathbf{5}$ & $\mathbf{1 0 0 , 0}$ \\
\hline
\end{tabular}

${ }^{*}$ Houve mais de uma resposta por sujeito. Percentual calculado pelo total de repostas.

Tabela 2 - Distribuição de frequência e percentual das dificuldades relacionadas à padronização do procedimento de cateterismo vesical, segundo instituições públicas, privadas e mista. Ribeirão Preto-SP, 2009

\begin{tabular}{|c|c|c|c|c|c|}
\hline \multirow{2}{*}{ Dificuldades } & \multicolumn{3}{|c|}{ Instituições } & \multicolumn{2}{|c|}{ Total } \\
\hline & Públicas & Privadas & Mista & $\mathbf{n}$ & $\%$ \\
\hline Não existe garantia de que o procedimento padronizado seja implementado & 3 & 5 & 1 & 9 & 27,4 \\
\hline Equipe médica não utiliza o protocolo estabelecido na instituição & 2 & 4 & 1 & 7 & 21,3 \\
\hline $\begin{array}{l}\text { Profissionais da enfermagem são capacitados de maneiras diferentes, nas } \\
\text { diferentes instituições onde trabalham }\end{array}$ & - & 3 & 1 & 4 & 12,1 \\
\hline Rotatividade dos profissionais da enfermagem & - & 3 & 1 & 4 & 12,1 \\
\hline Falta de tempo para treinamento da equipe & - & 3 & & 4 & 12,1 \\
\hline Indisponibilidade do material & - & 2 & & 2 & 6,0 \\
\hline Falta de supervisão e colaboração dos demais enfermeiros da instituição & 1 & - & & 1 & 3,0 \\
\hline Literatura diversificada sobre o assunto & - & - & 1 & 1 & 3,0 \\
\hline $\begin{array}{l}\text { Falta de mobilização dos setores para provimento dos materiais padroniza- } \\
\text { dos pela instituição }\end{array}$ & 1 & - & & 1 & 3,0 \\
\hline Total & 7 & 20 & 6 & 33 & 100,0 \\
\hline
\end{tabular}

${ }^{*}$ Houve mais de uma resposta por sujeito. Percentual calculado pelo total de repostas.

Dentre os fatores apresentados como positivos no processo de padronização, evidenciase a possibilidade de treinamento dos recursos humanos em enfermagem, o que reforça o uso da padronização dos procedimentos no treinamento da equipe, com a perspectiva de qualificar a assistência oferecida. No entanto, na instituição com financiamento misto, o entrevistado destaca a positividade do uso do cateter urinário pela comodidade tanto do paciente como da equipe de enfermagem, o que transgride os princípios de uma assistência segura ao paciente, confrontando com a literatura que correlaciona a inserção do cateter urinário como fator causal de riscos ao paciente. ${ }^{5-6,14,17-18}$

Quanto às dificuldades referidas à padronização do procedimento, observa-se que, em todas as instituições pesquisadas, o profissional entrevistado coloca em dúvida se o procedimento padronizado está sendo implementado, sendo também prevalente o fato de a equipe médica não utilizar o protocolo padronizado. Exclusivamente como dificuldades encontradas nas instituições privadas e mista são citadas diferentes capacitações reali- 
zadas sobre $\mathrm{o}$ assunto para os profissionais de enfermagem e a falta de tempo para treinamento. Os entrevistados destacam que uma vez que muitos profissionais da área de enfermagem trabalham em mais de uma instituição, em dupla jornada de trabalho, nem sempre seguem a padronização de um dos locais de trabalho, pois sentem insegurança pelas diferentes maneiras como aprendem o "como fazer" entre as instituições pesquisadas.

Almejando a qualidade do procedimento em estudo, as dificuldades encontradas reforçam a necessidade de maior integração entre as equipes de saúde e de enfermagem, internas e externas às instituições. Acresce-se ainda a necessidade do posicionamento do enfermeiro como líder da equipe, seja na gerência do setor onde atua, ou junto ao cuidado prestado nas diferentes instituições hospitalares do país, envolvendo-se efetivamente e provocando o envolvimento da equipe multiprofissional, reconstruindo com cientificidade o cuidado nas eliminações urinárias, num modelo de gestão que aperfeiçoe e aumente as probabilidades de sucesso e satisfação em saúde. Para tanto, acresce-se que ações direcionadas à educação permanente, utilizadas como instrumentos de qualificação da assistência de enfermagem, poderiam constituir-se em importantes ferramentas humanísticas a profissionais e pacientes. ${ }^{12,14,20}$

\section{CONCLUSÕES}

Embora as instituições estudadas apresentem o procedimento de cateterismo urinário padronizado, não existe a garantia de sua aplicação na prática assistencial, o que coloca em risco uma grande parcela de pacientes expostos a sua aplicação. O estudo demonstra ainda que diferenças no financiamento e nas características dos recursos humanos que sustentam as atividades e relações profissionais nas instituições podem gerar facilidades e dificuldades nas padronizações propostas.

Para tanto, sugere-se a implementação de ações interinstitucionais e interdisciplinares como estratégias a serem formuladas, tornandose facilitadoras na manutenção do treinamento e atividades da equipe.

A proposta de padronização do procedimento não proporciona garantias da sua realização, o que torna necessário avaliar se o procedimento proposto está sendo implementado e quais estratégias de treinamento são passíveis de aplicação na prática clínica para sustentar a atualização e a aplicação do conhecimento. Essas conclusões evidenciam a necessidade de investimento em pesquisas sobre a temática.

\section{REFERÊNCIAS}

1. Craven RF, Hirnle CJ. Fundamentos de enfermagem. Saúde e função Humanas [com] 37 colaboradores. $4^{\mathrm{a}}$ ed. Rio de Janeiro (RJ): Guanabara Koogan; 2006.

2. Potter PA, Perry AG. Fundamentos de enfermagem. $5^{a}$ ed. Rio de Janeiro (RJ): Guanabara Koogan; 2004.

3. Borba AMC, Lelis MAS, Brêtras ACP. Significado de ter incontinência urinária e ser incontinente na visão das mulheres. Texto Contexto Enferm. 2008 Jul-Set; 17(3):527-35.

4. Lenz LL. Cateterismo vesical: cuidados, complicações e medidas preventivas. Arquivos Catarinenses de Medicina. 2006; 35(1):82-91.

5. Joanna Briggs Institute (JBI). Removal of short-term indwelling urethral catheters. Best Pratice, Austrália. [página da Internet] 2006 [acesso 2009 Set 23]. Disponivel em http:/ / www.joannabriggs.edu.au/

6. Center for Disease Control and Prevention (CDC). Guideline for prevention of catheter-associated urinary tract infections, EUA. [página na Internet] 2009 [acesso 2010 Jan 02]. Disponível em: http:// www. cdc.gov/ncidod/dhqp/gl_catheter_assoc. htm.

7. Gagliardi EMDB, Fernandes AT, Cavalcante NJF. Infecção do trato urinário. In: Fernandes AT, editor. Infecção hospitalar e suas interfaces na área da saúde. São Paulo (SP): Atheneu; 2000. p.459-78.

8. Stamm AMNF, Forte DY, Sakamoto, KS, Campos, ML, Cipriano ZM. Cateterização vesical e infecção do trato urinário: estudo de 1092 casos. Arqu Catarin Med [online]. 2006 [acesso 2008 Jul 02]; 35(2). Disponível em: http://www.acm.org.br/revista/ pdf/artigos/361.pdf

9. Conselho Federal de Enfermagem - COFEN [página da internet]. Lei no 7.498, de 25 de junho de 1986. Dispõe sobre a Regulamentação do Exercício da Enfermagem. Ministério da Saúde. Brasília (DF): MS; 1986. [acesso 2008 Jul 01]; Disponível em: www. portalcofen.gov.br/2007/materiais.asp?Article.ID$22 \&$ sectionID $=35$.

10. Leaver RB. The evidence for urethral meatal cleansing. Nurs Stand. 2007 Jun; 21(41):39-42.

11. Alves MVMFF, Luppi CHB, Paker C. Condutas tomadas pelos enfermeiros relacionadas ao procedimento de sondagem vesical. Rev Cienc Ext. 2006 Dez; 3(1): 15.

12. Neto AQ. Segurança dos pacientes, profissionais e organizações: um novo padrão de assistência à saúde. Revist Adm Saúde [online] 2006 [acesso 2008 Jun 28]; 8(33). Disponível em: http:/ / www.cqh.org. br/files/RAS33-segurança.pdf 
13. Brasil. Normas e Padrões de Construções e Instalações de serviços de Saúde: Conceito e Definições - Hospital geral de pequeno e médio portes, unidades sanitárias. Brasília (DF): Ministério da Saúde; 1978.

14. Souza ACS, Tipple AFU, Barbosa JM, Pereira MS, Barreto, RASS. Cateterismo urinário: conhecimento e adesão ao controle de infecção pelos profissionais de enfermagem. Rev Eletr Enferm [online]. 2007 [acesso 2008 Jul 02]; 9(3). Disponível em: http:/ /www.fen. ufg.br/revista/v9/n3/v9n3a12htm

15. Guerrero GP, Beccaria LM, Trevizan MA. Procedimento operacional padrão: utilização na assistência de enfermagem em serviços hospitalares. Rev Latino-am Enfermagem [online]. 2008 [acesso 2009 Jul 13]; 16(33). Disponível em: http:/ /www. scielo.br/pdf/rlae/v16n6/pt_05.pdf

16. Organização Mundial de Saúde (OMS). Text of resolution WHA 55.18. Quality of care patiente safety-resolution WHA 55.18. [página na Internet]. 2002 [acesso 20 Jun 2008] Disponível em www.who. int/gb/ebwha/pdf-files/WHA55/ewha5518.pdf.

17. Sublett CM. Effect of time of day for urinary catheter removal on voiding behaviors in stroke patients the meaning of significance to the evidence base. Urologic Nursing. 2007; 2(3):236-38.

18. Ministério da Saúde (PT). Instituto Nacional de Saúde Dr. Ricardo Jorge. Recomendação para a prevenção da infecção do trato urinário. Agaliação de curta duração. Portugal: MS; 2004.

19. Ministério da Saúde (BR). Secretária de assistência à saúde. Manual Brasileiro de Acreditação Hospitalar. $5^{\mathrm{a}}$ ed. Brasília (DF): MS; 2006.

20. Rocha ESB, Trevizan MA. Gerenciamento da qualidade em um serviço de enfermagem hospitalar. Rev Latino-am Enfermagem [online] 2009 [acesso 2009 Ago 20]; 17(2). Disponível em: http:/ /www. scielo.br/pdf/rlae/v16n6/pt_05.pdf

E-mail: amazzo@eerp.usp.br 